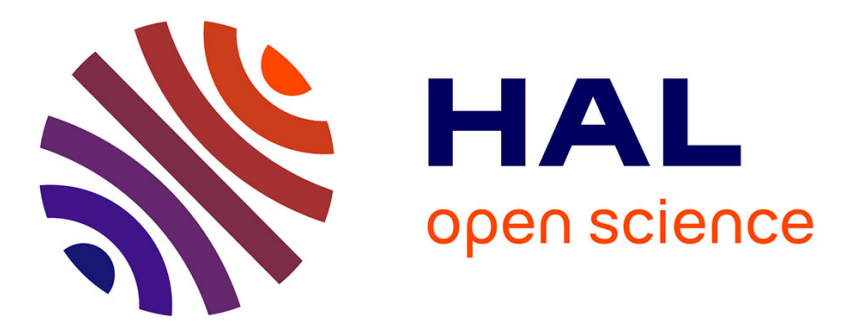

\title{
Terlipressin, a vasoactive prodrug recommended in hepatorenal syndrome, is an agonist of human V1, V2 and V1B receptors: Implications for its safety profile \\ Pascal Colson, Anne Virsolvy, Philippe Gaudard, Azzouz Charrabi, Maithé Corbani, Maxime Manière, Sylvain Richard, Gilles Guillon
}

\section{To cite this version:}

Pascal Colson, Anne Virsolvy, Philippe Gaudard, Azzouz Charrabi, Maithé Corbani, et al.. Terlipressin, a vasoactive prodrug recommended in hepatorenal syndrome, is an agonist of human V1, V2 and V1B receptors: Implications for its safety profile. Pharmacological Research, 2016, 113, pp.257 264. 10.1016/j.phrs.2016.08.027 . hal-01783524

\section{HAL Id: hal-01783524 \\ https://hal.umontpellier.fr/hal-01783524}

Submitted on 2 Dec 2019

HAL is a multi-disciplinary open access archive for the deposit and dissemination of scientific research documents, whether they are published or not. The documents may come from teaching and research institutions in France or abroad, or from public or private research centers.
L'archive ouverte pluridisciplinaire HAL, est destinée au dépôt et à la diffusion de documents scientifiques de niveau recherche, publiés ou non, émanant des établissements d'enseignement et de recherche français ou étrangers, des laboratoires publics ou privés. 


\title{
Terlipressin, a vasoactive prodrug recommended in hepatorenal syndrome, is an agonist of human V1, V2 and V1B receptors: Implications for its safety profile
}

\author{
Pascal H. Colson ${ }^{\mathrm{a}, \mathrm{c}, *, 1}$, Anne Virsolvy ${ }^{\mathrm{b}, 1}$, Philippe Gaudard ${ }^{\mathrm{b}, \mathrm{c}}$, Azzouz Charrabi ${ }^{\mathrm{b}}$, \\ Maithé Corbani ${ }^{\mathrm{a}}$, Maxime J. Manière ${ }^{\mathrm{a}}$, Sylvain Richard ${ }^{\mathrm{b}}$, Gilles Guillon ${ }^{\mathrm{a}}$ \\ a Institut de Génomique Fonctionnelle, Département d'Endocrinologie, CNRS UMR 5203, INSERM U1191, Université de Montpellier, F-34094 Montpellier, \\ France \\ b PhyMedExp, INSERM U1046, CNRS UMR 9214, Université de Montpellier, F-34295 Montpellier, France \\ ' Département d'Anesthésie Réanimation Arnaud de Villeneuve, Centre Hospitalier Régional et Universitaire, F-34295 Montpellier, France
}

Chemical compounds studied in the article:

Terlipressin, PubChem CID: 72081

[Arginine ${ }^{8}$ ]Vasopressin, PubChem CID:

90488786

[ Lysine $^{8}$ Vasopressin, PubChem CID:

90488785

SR49059, PubChem CID: 60943

Keywords:

V1A receptor

V2 receptor

Vasopressin receptors

Hepatorenal syndrome

Terlipressin

\begin{abstract}
A B S T R A C T
Terlipressin is recommended as a gold standard to treat hepatorenal syndrome complicating liver cirrhosis. It is presented as a specific V1A receptor agonist, beyond its enzymatic conversion into lysine ${ }^{8}$-Vasopressin (LVP), able to counteract the splanchnic vasodilation. However, the complete pharmacological characterization of this drug with respect to the different vasopressin receptor subtypes is missing. We studied terlipressin intrinsic properties, focusing not only on V1A, but also on other vasopressin receptor subtypes. The experimental studies were conducted on rat and human cellular models. Binding experiments were performed on rat liver membranes and $\mathrm{CHO}$ cells transfected with the different human vasopressin receptor subtypes. Agonist status was assessed from inositol phosphate or cyclic AMP assays, and measurement of intracellular calcium variations, performed on cultured vascular smooth muscle cells from rat aorta and human uterine artery and $\mathrm{CHO}$ cells. Terlipressin binds to the rat and human V1A receptors with an affinity in the micromolar range, a value 120 fold lower than that of LVP. It induces a rapid and transient intracellular calcium increase, a robust stimulation of phospholipase $\mathrm{C}$ but with reduced maximal efficiencies as compared to LVP, indicating a partial V1A agonist property. In addition, terlipressin is also a full agonist of human V2 and V1B receptors, with also a micromomolar affinity.

Conclusions: Terlipressin is a non-selective vasopressin analogue, exhibiting intrinsic agonist properties. Its full V2 receptor agonism may result in renal effects potentially aggravating water retention and hyponatremia of cirrhosis.
\end{abstract}

Abbreviations: AVP, [arginine ${ }^{8}$ ]vasopressin; LVP, [lysine ${ }^{8}$ ]vasopressin; HRS, hepatorenal syndrome; VSMCs, vascular smooth muscle cells; hV1A-R, hV1B-R and hV2-R, human V1A V1B and V2 vasopressin receptor; BSA, bovine serum albumin; PLC, phospholipase C; IPs, total inositol lipids; AC, adenylate cyclase; ATP, adenosyl triphospshate; cAMP, cyclic adenosyl monophosphate; $\left[\mathrm{Ca}^{2+}\right]_{1}$, cytosolic $\mathrm{Ca}^{2+} ; \mathrm{K}_{\mathrm{i}}$, affinity constant of peptide; $\mathrm{K}_{\mathrm{act}}$, concentration of peptide leading to half maximal stimulation; $\mathrm{E}_{\max }$, peptide maximal efficiency.

* Corresponding author at: Service d'anesthésie-réanimation "ADV", Centre Hospitalier Universitaire de Montpellier, 371 avenue Doyen Giraud, 34295, Montpellier Cedex 5, France.

E-mail addresses: p-colson@chu-montpellier.fr (P.H.Colson), anne.virsolvy@inserm.fr (A.Virsolvy), p-gaudard@chu-montpellier.fr (P. Gaudard), azzouz.charrabi@inserm.fr (A. Charrabi), maithe.corbani@igf.cnrs.fr (M. Corbani), m.maniere@gmail.com (M.J. Manière), sylvain.richard@inserm.fr (S. Richard), gilles.guillon@igf.cnrs.fr (G. Guillon).

${ }^{1}$ These authors contributed equally to the manuscript.

\section{Introduction}

Terlipressin is currently the treatment of reference for patients with hepatorenal syndrome (HRS) complicating liver cirrhosis, especially for HRS type 1 a particular form with acute functional renal failure due to splanchnic and systemic vasodilatation $[1,2]$. Terlipressin is a synthetic peptide composed of one molecule of $\left[\right.$ Lysine $^{8}$ ]Vasopressin (LVP) and three additional glycine residues associated to its $\mathrm{N}$-terminal end. It is metabolized via exopeptidases into LVP, the natural vasopressin porcine hormone. Terlipressin is presented as an inactive pro-drug able to release sustained amounts of LVP, the active form providing prolonged biological effect $[3,4]$. The vasoactive properties of LVP decreases splanchnic blood flow in order to prevent oesophageal varices haemorrhage or the hepatorenal syndrome complicating liver cirrhosis [5-8]. In this specific indication, terlipressin may improve renal perfusion and may pro- 
long survival. It is considered as a standard of care in Europe [9]. Recent studies suggest that its beneficial effect may be extended in case of HRS associated with sepsis [10].

The terlipressin vasopressor effects are thought to result from the slow enzymatic conversion into LVP and a potentiation of adrenergic vasoconstriction [11]. However, some studies suggest that terlipressin exhibit intrinsic vasoconstrictive properties [12]. In a previous report [13], we have shown that terlipressin triggers a strong vasocontriction of both rat and human arteries via the activation of V1A receptor. This effect develops quickly, suggesting that terlipressin, besides its pro-LVP status, could also be considered as a direct vasoconstrictor drug per se. Terlipressin is presented as a V1A receptor agonist [14], but it is known that vasopressin analogues and especially LVP could interact with other receptor subtypes (V1B and V2). If a direct effect of terlipressin on arteries has been evidenced, the complete pharmacological properties of terlipressin for the different human vasopressin receptor subtypes have never been established so far.

The aim of this work was to characterize the full intrinsic pharmacological properties of terlipressin for all the human vasopressin receptor subtypes. Improved knowledge of these pharmacological properties is necessary to understand complete mode of action of the drug and to unveil possible therapeutic limitations.

\section{Materials and methods}

\subsection{Animals and human tissues}

Male Wistar rats (225-250g) were provided by Janvier (Le Genest-St-Isle, France). Experiments were conducted in accordance with national law and approved by the committee on animal care of Montpellier-Languedoc-Roussillon (No. CEEA-LR-12075).

Human uterine arteries were obtained from patients undergoing hysterectomy. The procedures were approved by national ethic Committee and the French Ministry of Research (DC-2008-488) and patients gave informed consent.

\subsection{Smooth muscle cell isolation and culture}

Vascular smooth muscle cells (VSMCs) were isolated from rat aorta and from human uterine artery. The descending thoracic aorta was collected from six week-old male Wistar rats anesthetized with intra-peritoneal pentobarbital $(1.5 \mathrm{ml} / \mathrm{kg})$ and uterine arteries were excised from the parametrium, connective tissues and adjacent myometrium immediately after surgery. Arteries were aseptically immersed in isolation isotonic buffer and media layers were dissected. Cells were enzymatically isolated and grown in primary culture as previously described [15]. Cells were used during 5 passages.

\subsection{Analysis of terlipressin purity}

Purity of terlipressin was evaluated by mass spectrometry as previously described [16] using an UltraFlex TOF/TOF mass spectrometer (Bruker-Daltonik, Bremen, Germany) The sensibility of the MALDI-TOF spectrometer used in the study was about $1 \mathrm{fem}-$ tomole.

\subsection{Binding experiments}

The affinity of terlipressin was determined by competition binding experiments using $\left[{ }^{3} \mathrm{H}\right] \mathrm{AVP}$ as radioligand on two biological models: rat liver membranes known to naturally express high level of V1A vasopressin receptor isoform and membranes from CHO cells stably transfected with the human V1A, V1 B and V2 receptor (hV1A-R, hV1B-R, and hV2-R, respectively) as previously described [17]. Note that our experimental conditions included $0.1 \%$ Bovine Serum Albumin (BSA) and Leupeptin $(0.1 \mathrm{mg} / \mathrm{ml})$ preventing noticeable terlipressin conversion into LVP.

\subsection{Second messenger measurements}

The ability of terlipressin to stimulate Phospholipase C (hV1A$\mathrm{R}, \mathrm{hV1B}-\mathrm{R}$ ) or Adenylate Cyclase activities (hV2-R) was tested on rat and human cultured VSMCs naturally expressing V1A-R and on CHO cell transfected and stably expressing hV1A-R or hV2-R.

Phospholipase C (PLC) activity was assessed as described previously [17] by labelling cellular Phosphoinositol lipids during preincubation with myo- $\left[2-{ }^{3} \mathrm{H}\right]$ inositol, then measuring total inositol phosphates (IPs) generated under hormonal stimulation.

Adenylate cyclase (AC) activity was assessed as described previously [18] by labelling intracellular Adenosyl Triphosphate (ATP) by pre incubation with $\left[{ }^{3} \mathrm{H}\right]$ Adenine, then measuring cyclic Adenosyl Monophosphate (CAMP) generated under hormonal stimulation.

\subsection{Intracellular calcium variation measurements}

Changes in cytosolic $\mathrm{Ca}^{2+}\left(\left[\mathrm{Ca}^{2+}\right]_{\mathrm{i}}\right)$ were measured using the ratiometric fluorescent $\mathrm{Ca}^{2+}$ indicator Fura- 2 as previously described [19]. Results were normalized to the effect of $100 \mathrm{nM}$ LVP. The changes in $\left[\mathrm{Ca}^{2+}\right]_{\mathrm{i}}$ were deduced from the variations of the fluorescence ratio after correction for background and dark currents. Data were averaged (at least 20-25 cells per recorded field/coverglass). One field, chosen randomly, was recorded in each coverglass, $\mathrm{n}$ represented the number of cell cultures.

\subsection{Chemicals}

Commercial terlipressin was obtained from Ferring A. B. (Limhamn, Sweden), Arginine ${ }^{8}$ Vasopressin (AVP) and LVP from Bachem (Bubendorf, Switzerland) and SR49059 from SanofiAventis (Sanofi-Aventis, Toulouse, France). Most standard chemicals were purchased from Sigma (St Louis, MO), Roche Molecular Biochemicals (Mannheim, Germany) or Merck and Co (Darmstadt, Germany) unless otherwise indicated. $\left[{ }^{3} \mathrm{H}\right] \mathrm{AVP}(60-80 \mathrm{Ci} / \mathrm{mmol})$, myo- $\left[2-{ }^{3} \mathrm{H}\right]$ inositol $(10-20 \mathrm{Ci} / \mathrm{mmol})$ were obtained from PerkinElmer Life Sciences (Courtaboeuf, France).

\subsection{Data analysis}

The radioligand binding data were analyzed by GraphPad Prism (GraphPad Software, Inc., San Diego, CA) as previously described [18]. Results are expressed as mean \pm SEM. Statistic analysis consisted in ANOVA and Student's $t$-test, and p value $<0.05$ considered as statistically significant.

\section{Results}

\subsection{Terlipressin purity}

The mass spectrum obtained for 0.1 pmole terlipressin showed a major peak at a molecular mass of 1227 that corresponded to the theoretical mass of terlipressin and of two minor peaks at molecular mass corresponding respectively to terlipressin associated with one $\mathrm{Na}^{+}$cation and one $\mathrm{K}^{+}$cation (1250 and 1256 respectively) (Fig. 1 panel A). Analysis of this spectrum also revealed the presence of other peaks of very low amplitude and lower molecular mass. According to their molecular weight, these peaks corresponded to shorter forms of terlipressin respectively deprived of 1-3 glycine residues: desGly1-terlipressin, desGly2-terlipressin and desGly3terlipressin representing LVP (Fig. 1 panel B). 


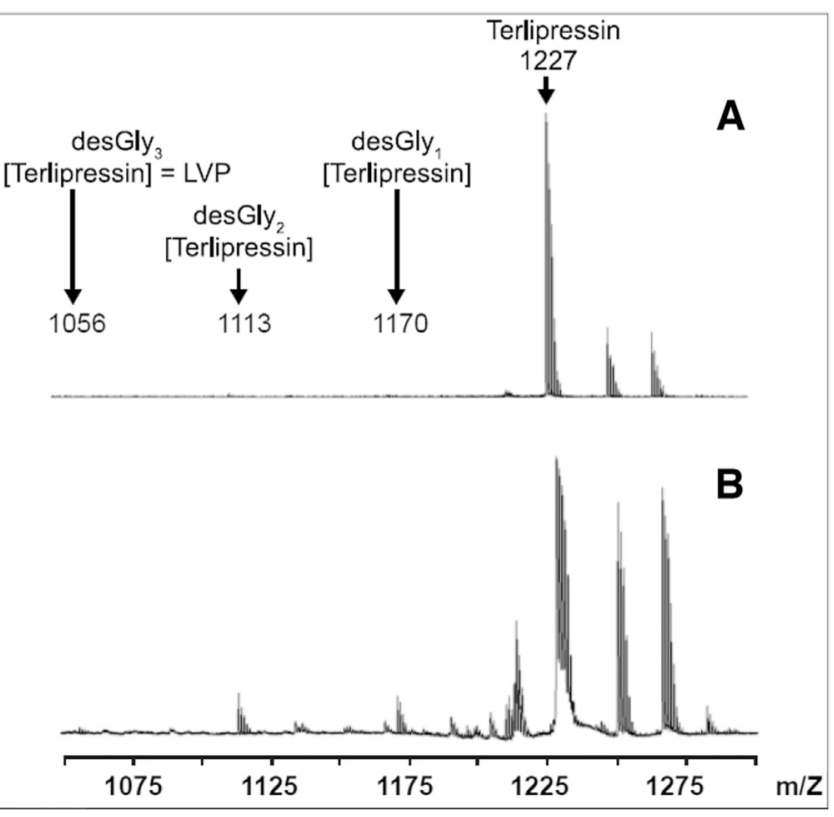

Fig. 1. Mass spectrometry of commercial terlipressin.

Mass spectrum for 0.1 (panel A) or 1 pmole terlipressin (panel B) was performed as described under methods. The mass spectrometer was calibrated with known amounts of AVP and the mass spectrum was corrected considering the different desorption rates of each peptide studied. AVP exhibiting the same "tocin ring" as LVP and terlipressin was thus used as an external standard. Terlipressin deprived of 1-3 glycine residues results in desGly1-terlipressin, desGly2-terlipressin and desGly3terlipressin (representing LVP), respectively. Results illustrated are representative of 3 distinct experiments and showed that terlipressin used in this study is extremely pure.

Quantitative analysis was further performed to determine the rate of LVP contaminating terlipressin samples. We found that the amount of LVP present in our terlipressin stock solution represented a ratio of $1.3 \pm 0.3 \%$ of terlipressin ( 3 independent determinations).

\subsection{Affinity of terlipressin for vasopressin receptors}

\subsubsection{Rat and human V1A receptor}

AVP and LVP dose-dependently inhibited $\left[{ }^{3} \mathrm{H}\right]$ AVP specific binding whatever the species considered (Fig. 2, left upper panels). As expected, the affinity of $\operatorname{LPP}\left(K_{i}\right)$ was $2-3$ fold weaker as compared to AVP for both rat and human V1A receptors (Table 1). Similarly, terlipressin dose-dependently inhibited $\left[{ }^{3} \mathrm{H}\right] \mathrm{AVP}$ specific binding. However, when compared to LVP, the affinity of terlipressin was reduced by 122 and 710 folds respectively for the rat and human V1A receptors respectively (Table 1 ).

\subsubsection{Other human vasopressin receptors}

LVP non-selectively interacted with all the vasopressin receptor isoforms with a nanomolar affinity $\left(\mathrm{K}_{\mathrm{i}}\right.$ in $\mathrm{nM}$ : $1.2 \pm 0.2$ for hV1A$\mathrm{R} ; 1.4 \pm 0.3$ for hV1B-R; $0.8 \pm 0.1$ for hV2-R). Terlipressin also nonselectively interacted with all the vasopressin receptors but with an affinity reduced by at least 710 folds ( $\mathrm{K}_{\mathrm{i}}$ in $\mathrm{nM}: 852 \pm 71$ for hV1A$\mathrm{R} ; 1115 \pm 70$ for hV1B-R; and $1580 \pm 50$ for hV2-R) (Fig. 2, left lower panels).

\subsection{Effects of terlipressin on second messenger pathways}

3.3.1. On rat and human arterial smooth muscle cells naturally expressing $V 1 \mathrm{~A}$ receptors

On human cultured VSMCs, LVP and AVP dose-dependently stimulated PLC activity to the same maximal extend with a nanomolar $K_{\text {act }}$ (concentration of peptide leading to half maximal stimulation) (Fig. 2, right upper panel, Table 1). Terlipressin was less efficient as compared to LVP and its maximal $\mathrm{IP}_{\mathrm{s}}$ production $\left(E_{\max }\right)$ represented only $50 \pm 4 \%$ of the LVP $E_{\max }$. Additionally, its $\mathrm{K}_{\mathrm{act}}$ was 124 fold higher when compared to LVP.

Similar results were obtained with rat VSMCs. Terlipressin was less active than LVP, PLC stimulation represented $29 \%$ of LVP $E_{\max }$ and $K_{\text {act }}$ value was higher than that of LVP (Fig. 2 right upper panel, Table 1).

In the presence of protease inhibitors (bestatin and BSA) used to prevent terlipressin conversion into LVP, no significant changes were observed for a short incubation-period ( $2 \mathrm{~min}$ ) in either terlipressin or LVP IPs accumulation (Fig. 3, upper panels). After a $30 \mathrm{~min}$ incubation period, terlipressin-induced accumulation of IPs was reduced in the presence of BSA and bestatin, but not suppressed. In the same conditions, no changes were noticed for LVP. Similar results were obtained on rat VSMCs (data not shown).

A pre-incubation with the selective V1A antagonist SR49059 (100 nM) completely abolished the stimulatory effects of LVP on PLC activity either for a 2-min or a 30-min incubation period. Such pre-incubation also completely inhibited terlipressin-stimulated PLC activity (Fig. 3, lower panels). Similar results were obtained on rat VSMCs (data not shown).

\subsubsection{On heterologous cellular models stably transfected with human VP receptors}

We also evaluated the effect of terlipressin on PLC or adenylate cyclase (AC) activities in $\mathrm{CHO}$ cells stably transfected with the different human vasopressin receptor subtypes (hV1A-R, hV1B-R or hV2-R). LVP dose-dependently stimulated IPs or CAMP productions, validating a functional coupling with PLC and AC activities in these cells (Fig. 2, right lower panels). We observed that terlipressin similarly induced a dose-dependent increase in IPs or cAMP productions whatever the receptor subtype considered. It also behaved as an almost full agonist of hV2-R $\left(E_{\max }=89 \pm 3 \%\right.$ of that of LVP $\left.E_{\max } ; K_{a c t}=1570 \pm 260 \mathrm{nM}\right)$ and hV1B-R $\left(E_{\max }=91 \pm 3 \%\right.$ of that of $L_{V P} E_{\max } ; K_{a c t}=717 \pm 59 \mathrm{nM}$ ) (Fig. 2, right lower panels).

As a control, we also observed in the $\mathrm{CHO}$ model transfected with $\mathrm{hV} 1 \mathrm{~A}-\mathrm{R}$, the partial agonist properties of terlipressin that we found in cultured VSMCs naturally expressing V1A-R $\left(E_{\max }=73 \pm 2 \%\right.$ of LVP $E_{\max } ; K_{a c t}=310 \pm 53 \mathrm{nM}$ ).

\subsection{Terlipressin effects on intracellular calcium in arterial smooth muscle cells}

AVP and LVP both induced dose-dependent and transient increase in $\left[\mathrm{Ca}^{2+}\right]_{\mathrm{i}}$ in rat and human cultured VSMCs (Fig. 4, upper panels). For each agonist, $K_{a c t}$ value was in the nanomolar range (Table 1). AVP, with the best affinity, presented the lower $K_{a c t}$ value and the higher maximal efficiency both in rat and human VSMCs. As expected, LVP displayed the same maximal efficiency as AVP. We observed that terlipressin also induced $\left[\mathrm{Ca}^{2+}\right]_{\mathrm{i}}$ increase (Fig. 4, upper panels). Its $K_{\text {act }}$ value, in the micromolar range, was higher than that of AVP and LVP. Its maximal efficiency was lower as compared to those of AVP and LVP and corresponded to $48 \pm 5 \%$ and $65.7 \pm 2 \%$ of the maximal $\left[\mathrm{Ca}^{2+}\right]_{\mathrm{i}}$ increase induced by LVP in rat and human VSMCs respectively (Table 1 ).

Time course variations of $\left[\mathrm{Ca}^{2+}\right]_{i}$ (Fig. 4, lower panels) compared the temporal response to terlipressin, LVP and AVP. Whatever the peptide used at a concentration allowing a maximal receptor occupancy, all recordings revealed a rapid and transient increase in $\left[\mathrm{Ca}^{2+}\right]_{i}$ with a peak between $22 \mathrm{~s}$ and $28 \mathrm{~s}$ after drug application both in rat and human VSMCs. 


\section{$(\Delta)$ Terlipressin \\ (O) AVP \\ (口) LVP}

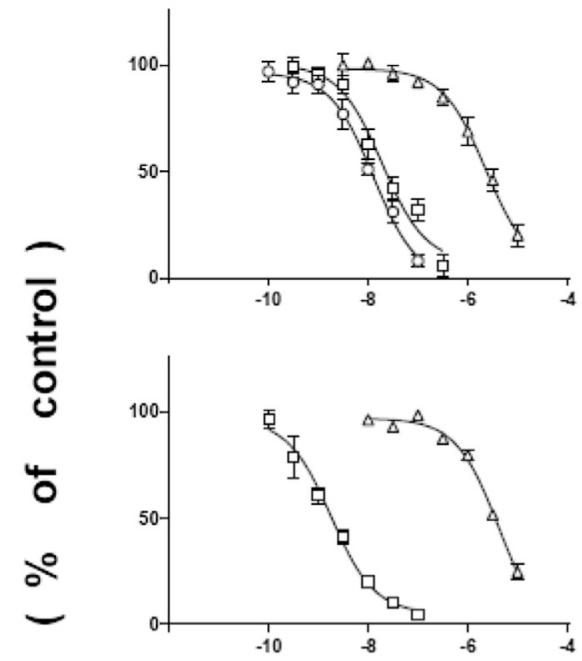

ติ
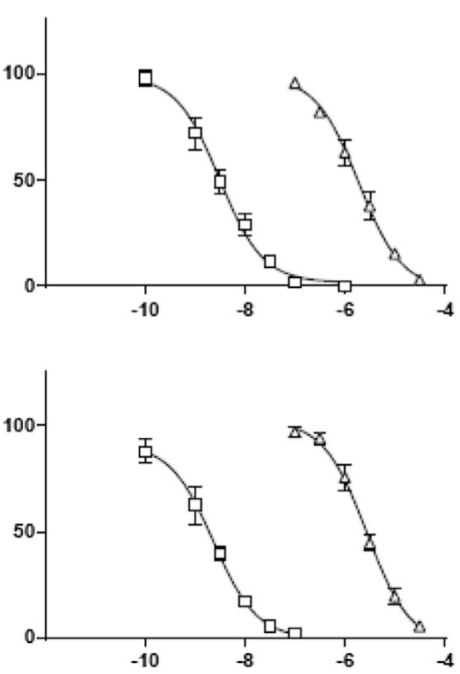

Agonist ( $\log [\mathrm{M}])$
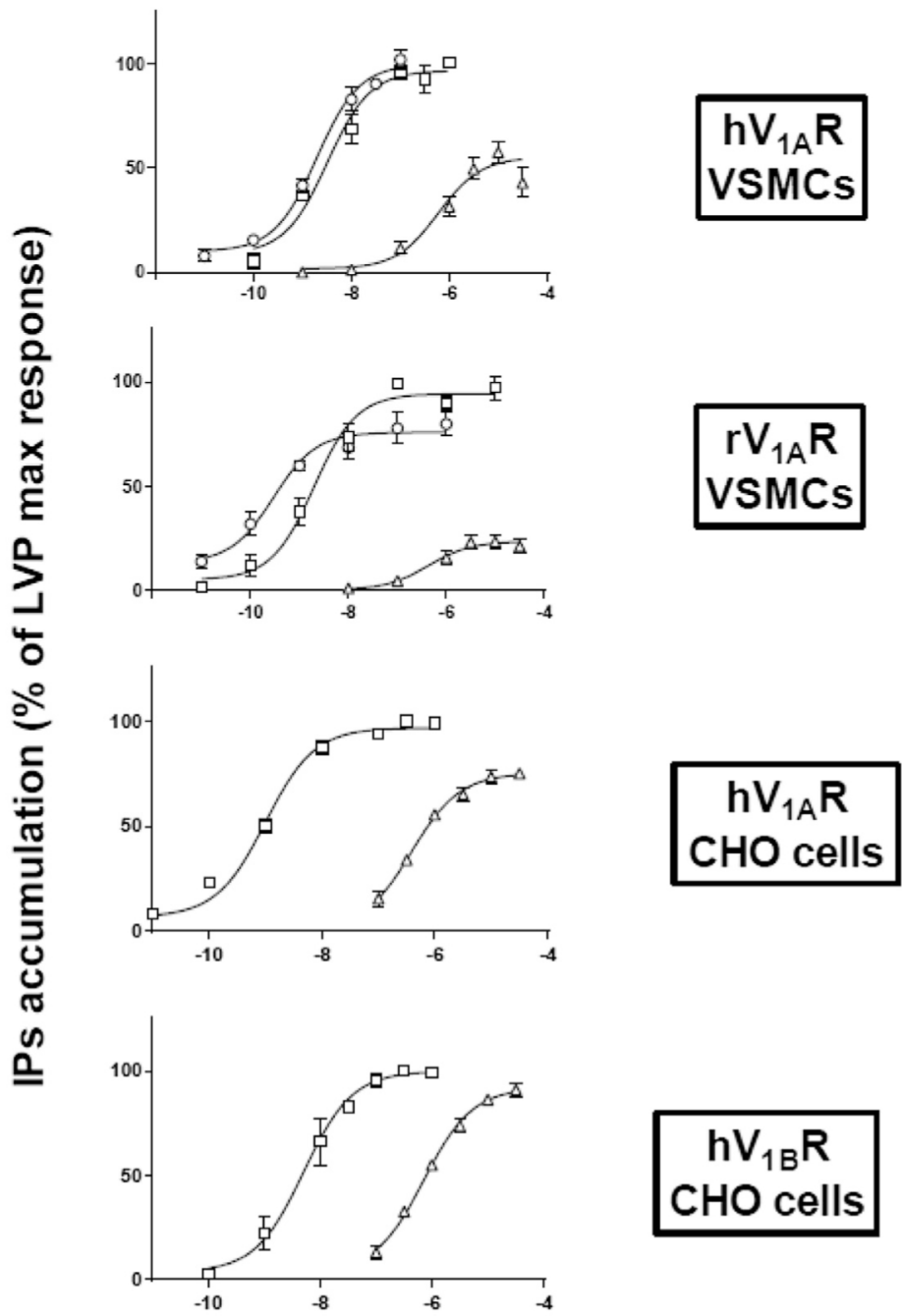

\section{$h V_{1 B} R$ \\ CHO cells}

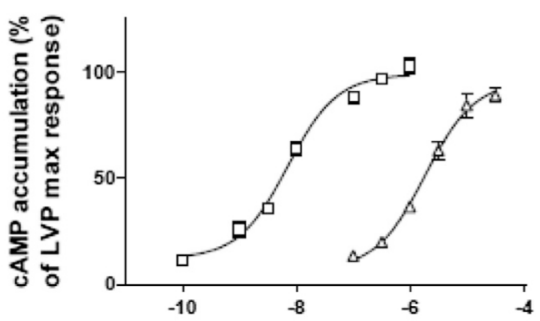

Agonist $(\log [\mathrm{M}])$

Fig. 2. Binding and functional properties of AVP, LVP and terlipressin for the rat and human vasopressin/oxytocin receptors.

Left panels: The affinity of terlipressin, AVP and LVP for the rat and human vasopressin/oxytocin receptors were determined by competition binding experiments using [ $\left.{ }^{3} \mathrm{H}\right] \mathrm{AVP}$ as radioligand (see methods). For the rat V1A-R (upper panel), experiments were performed on rat liver plasma membrane naturally expressing this receptor isoform. For the human receptors (lower panels), experiments were performed on membranes prepared from CHO cells stably transfected with the different human vasopressin receptor isoforms. Results are expressed as\% of specific binding measured in the absence of unlabeled hormone. Note that binding experiments were performed in the presence of leupeptin and BSA to prevent from terlipressin degradation into LVP.

Rights panels: The ability of terlipressin, AVP and LVP to stimulate phospholipase $\mathrm{C}$ was tested on human uterine artery and rat aortic cultured VSMCs (upper panels) by measuring total IPs which accumulated in response to hormonal stimulation (see methods). Results were expressed as\% of maximal LVP stimulation ( $100 \%=8.0 \pm 0.7$ and $6.7 \pm 0.7$ fold stimulation over control for respectively rat and human VSMCs). For Terlipressin, maximal stimulation expressed in fold of stimulation over control are $2.3 \pm 0.2$ and $3.3 \pm 0.3$ for respectively rat and human VSMCs. Maximal LVP and terlipressin responses are significantly different for both rat and human cells ( $\mathrm{P}<0.001$ ). Similar experiments were performed on CHO cells stably transfected with the hV1A-R, hV1B-R (middle panels) known to be positively coupled to PLC activation and IPs production. For the hV2-R known to stimulate adenylate cyclase (lower panel), cAMP accumulation was determined as described under methods and results were expressed as\% of maximal LVP responses $(100 \%=23 \pm 2$ fold stimulation over control)

Results are the mean \pm SEM of at least 3 distinct experiments each performed in triplicate. They shown that terlipressin is able to bind to rat and human V1A-receptors and to stimulate IPs production yet with less efficiency as compared to LVP or AVP. 
Functional properties of Terlipressin and natural hormones on rat and human aortic cells in primary culture.

\begin{tabular}{|c|c|c|c|c|c|c|c|}
\hline \multirow[t]{2}{*}{ Pharmacological properties } & \multirow[b]{2}{*}{ Rat } & \multicolumn{2}{|l|}{ LVP } & \multicolumn{2}{|l|}{ Terlipressin } & \multicolumn{2}{|l|}{ AVP } \\
\hline & & human & Rat & human & Rat & human & \\
\hline Affinity $K_{i}(n M)$ & & $10.1 \pm 2.5(5)$ & $1.2 \pm 0.2(5)$ & $1229 \pm 133(5)$ & $852 \pm 71(3)$ & $3.10 \pm 0.11(3)$ & $0.56 \pm 0.05(4)$ \\
\hline \multirow{2}{*}{ Phospholipase $C$ activity } & $\mathrm{K}_{\mathrm{act}}(\mathrm{nM})$ & $1.8 \pm 0.6(6)$ & $2.9 \pm 0.7(3)$ & $590 \pm 70(5)$ & $361 \pm 49(3)$ & $0.20 \pm 0.09(3)$ & $0.90 \pm 0.10(3)$ \\
\hline & $\mathrm{E}_{\max }(\%)$ & $100(12)$ & $100(10)$ & $29 \pm 3(17)$ & $50 \pm 4(9)$ & $97 \pm 7(6)$ & $97 \pm 2(3)$ \\
\hline \multirow[t]{2}{*}{ Intracellular calcium mobilization } & $\mathrm{K}_{\mathrm{act}}(\mathrm{nM})$ & $20.8 \pm 4.9(6)$ & $12 \pm 1.8(6)$ & $1400 \pm 180(8)$ & $942 \pm 240(8)$ & $3.2 \pm 1.3(6)$ & $7.16 \pm 1.60(6)$ \\
\hline & $\mathrm{E}_{\max }(\%)$ & $100(6)$ & $100(6)$ & $48 \pm 5(8)$ & $66 \pm 3(8)$ & $109 \pm 6(6)$ & $98 \pm 2(6)$ \\
\hline
\end{tabular}

Experiments were conducted as described in methods. Affinity $\left(\mathrm{K}_{\mathrm{i}}\right)$ for each analogue was determined by competition binding experiments as illustrated Fig. 2 . $\mathrm{K}_{\text {act }}$ were determined from experiments illustrated Figs. 2 and 4. $E_{\max }$ (maximal stimulation of a given analogue expressed in\% of maximal LVP response) were determined from experiments illustrated Figs. 2 and 4. Results are mean \pm SEM for the number of independent experiments indicated under parentheses, each performed in triplicate. These data show that terlipressin binds to V1A-R with a bad affinity as compared to LVP whatever the species considered and behaves as a partial V1A agonist whatever the assay used.

\section{Discussion}

In this study, we characterized the agonist properties of terlipressin for the different vasopressin receptor subtypes on rat and human cellular models. Beyond its enzymatic conversion into LVP, terlipressin exhibited intrinsic properties and was found to be a partial agonist for V1A and a full agonist for V1 B and V2 receptors.

\subsection{Terlipressin purity}

Terlipressin provided by Ferring Laboratories was a pure (triglycyl-LVP) peptide although we detected a slight contamination by LVP $(1.3 \pm 0.3 \%$ ). This contamination cannot explain the observed pharmacological effects of terlipressin since, when used at concentrations allowing full receptor occupancy, terlipressin and LVP would have the same maximal efficiency $\left(\mathrm{E}_{\max }\right)$ both on IPs accumulation and $\left[\mathrm{Ca}^{2+}\right]_{i}$ increase as LVP. We determined that terlipressin was less efficient than LVP in our experimental models.

\subsection{Terlipressin effect on V1A receptor}

The pharmacological properties of terlipressin described in this study are those of the native molecule. Indeed, preventing terlipressin conversion into LVP with protease inhibitors and BSA did not suppress receptor binding and second messenger production. Terlipressin was able to directly interact with the V1A-R with an affinity at least 100 fold lower than LVP and 400 fold lower than AVP.

As a consequence, terlipressin was able to directly stimulate IPs production, illustrating the functional coupling with PLC activity [20]. Like AVP and LVP [21], the capacity of terlipressin to increase IPs production was in close relationship with its binding properties. However, terlipressin $\mathrm{E}_{\max }$ for the hV1A-R expressed in VSMCs was only $50 \%$ of the LVP or AVP $E_{\max }$, clearly indicating that terlipressin behaves as a partial V1A agonist. Rationally, this partial V1A agonist property is corroborated by terlipressin induced $\left[\mathrm{Ca}^{2+}\right]_{\mathrm{i}}$ increase, with a $\mathrm{E}_{\max }$ lower than those of LVP and AVP.

Using rat and human cells naturally expressing V1A-R, we observed that both the affinity $\left(\mathrm{K}_{\mathrm{i}}\right)$ and the efficacy $\left(\mathrm{K}_{\mathrm{act}}\right)$ of terlipressin for V1A receptor were better for the human form. Moreover, terlipressin was also more efficient in human cells regarding IPs accumulation and $\left[\mathrm{Ca}^{2+}\right]_{\mathrm{i}}$ increase. Such observations are in line with the pharmacological differences previously reported between species for the vasopressin receptor family [22]. In human, both the higher affinity for the hV1A-R and the better functional efficiency may increase the activity of terlipressin in vivo. These results are consistent with our previous report on the vascular effects of terlipressin, in which we observed a more potent effect of terlipressin on human vessels than on rat vessels [13].

Although terlipressin is a partial agonist for the hV1A-R as compared to LVP or AVP, plasma peak concentration resulting from intravenous bolus administration of $1-2 \mathrm{mg}$ terlipressin seems however sufficient to induce a rapid vasoconstriction [13]. Moreover, an amplification loop observed for vasopressin receptors in many cellular systems may contribute to increase the sensitivity of vascular beds [11,23], with a high ischemia risk [9]. Indeed, some concerns have been raised from observation of myocardial ischemia shortly after intravenous bolus administration of terlipressin that may be accounted for by terlipressin-induced coronary vasoconstriction [24]. Similarly, in the context of HRS and compared to intravenous boluses, continuous intravenous infusion of terlipressin is associated with fewer adverse events, including bowel ischemia, which further suggests a direct deleterious effect of terlipressin before its conversion into LVP [25].

\subsection{Terlipressin effect on other vasopressin receptors}

This study provides the complete characterization of terlipressin pharmacological properties for all human vasopressin receptor isoforms including hV2-R and hV1B-R over and above hV1A-R.

Despite of a lower affinity for the hV2-R, terlipressin behaved nearly as a full agonist of this receptor isoform and was almost as efficient as AVP or LVP to stimulate AC activity. Previous reports advocated the fact that terlipressin is more specific for hV1A-R as compared to hV2-R $[26,27]$. Our data show that there is only a weak binding selectivity, with a hV1A-R/hV2-R selectivity ratio of 1.9. Therefore, terlipressin specificity for hV1A-R must be ruled out. We show that terlipressin triggers a sustained activation of hV2-R known to be involved in multiple regulations including vasodilatation, water retention, hyponatremia and coagulation. Hyponatremia has been reported previously with terlipressin treatment for severe variceal bleeding, and it was sometimes serious enough to cause threatening neurological consequences [28]. A terlipressin V2 effect has been incriminated through aquaporinmediated water transport [29]. However, in these clinical studies, it is difficult to assess whether the effect is directly related to terlipressin or secondary to its conversion into LVP. Our results confirm that a direct effect of terlipressin is possible, probably more likely when terlipressin is administered by intravenous boluses.

Similarly, the pharmacological characteristics observed for the hV1B-R revealed that terlipressin behaves as an almost full hV1B agonist of low affinity. This result suggests that, in vivo, terlipressin may also induce ACTH-stimulated cortisol release [30]. The hypothalamic-pituitary-adrenal axis can affect renal $\mathrm{Na}^{+}$homeostasis. Glucocorticoid can stimulate renal transport directly or indirectly in a very complex, not definitively established process [31]. These actions may be clinically relevant and may contribute to counteract the V2 effect. 

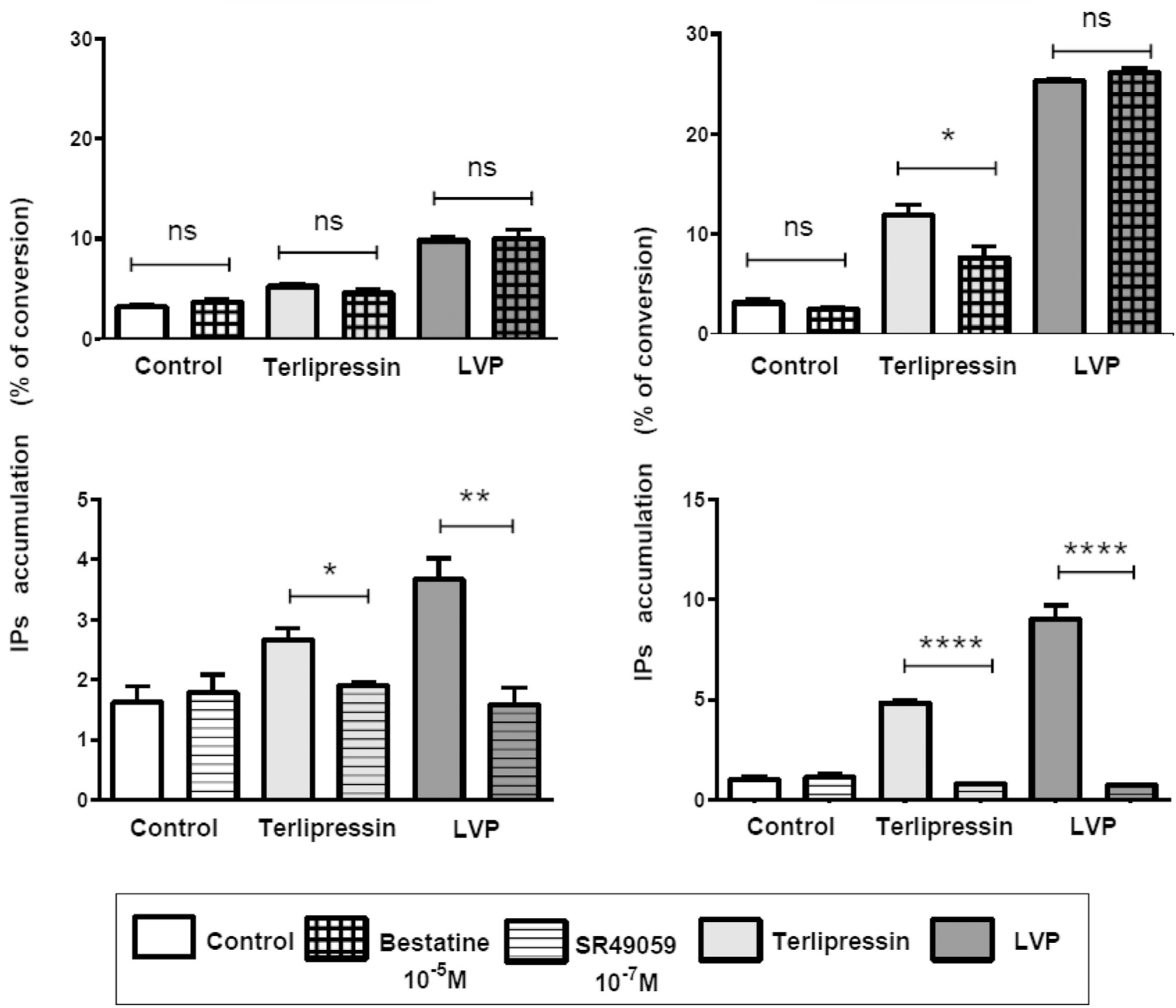

Fig. 3. Effect of a V1A antagonist and proteolytic conditions on LVP or terlipressin-induced stimulation of Phospholipase C activity in cultured human uterine artery smooth muscle cells.

Cultured human uterine artery and cultured smooth muscle cells were loaded with myo-[2- $\left.{ }^{3} \mathrm{H}\right]$ inositol, and inositol phosphate accumulation assay was performed after incubation in various conditions as described in methods.

Upper panels: Cells were pre-incubated $20 \mathrm{~min}$ at $37^{\circ} \mathrm{C}$ in the presence of $\mathrm{LiCl}\left(2.10^{-2} \mathrm{M}\right)$ with the protease inhibitor Bestatin $\left(10^{-5} \mathrm{M}\right)$ and BSA $(0.1 \%)$, conditions known to reduce peptide degradation and thus terlipressin conversion into LVP. Cells were further incubated in the same media for 2 or 30 additional min in the presence of terlipressin $\left(10^{-5} \mathrm{M}\right)$, LVP $\left(10^{-6} \mathrm{M}\right)$ or with vehicle (control).

Lower panels: Cells were pre-incubated $20 \mathrm{~min}$ at $37^{\circ} \mathrm{C}$ in the presence of $\mathrm{LiCl}\left(2.10^{-2} \mathrm{M}\right)$ with or without SR49059 $\left(10^{-7} \mathrm{M}\right)$ and further incubated for 2 or $30 \mathrm{~min}$ in the presence of Terlipressin $\left(3.1610^{-7} \mathrm{M}\right)$, LVP $\left(10^{-8} \mathrm{M}\right)$ or with vehicle (control). Total Inositol phosphates (IPs) that accumulated and total Phosphoinositol lipids (PIs) remaining in the cells were determined for each sample. Results are expressed as\% of PIs converted into total IPs.

Results are mean \pm SEM for one representative experiment of three, each performed in quadruplicate with *, $\mathrm{P}<0.05$; **, $\mathrm{P}<0.01$; ***, $\mathrm{P}<0.0001$. They demonstrated that terlipressin interacts with V1A receptors to trigger PLC activation and that its conversion in LVP is not necessary to stimulate IPs production.

\subsection{Vasopressin agonist and HRS}

Our results show that terlipressin is a partial agonist for hV1A$\mathrm{R}$, and a full agonist for hV2-R and hV1B-R. These characteristics cannot support the usual concept of terlipressin hV1A selectivity.

Therefore, the expected effects of terlipressin are those of AVP or LVP. Beside the beneficial reversing effect on splanchnic vasodilation, terlipressin could therefore paradoxically contribute to aggravate the cirrhosis pathology, inducing more water retention and hyponatremia, through the activation of the V2-R [32]. Conversely, especially in the case of HRS with sepsis, vasopressin treatment might improve cortisol production, a potential bene- fit owing to the associated adrenal insufficiency [33]. However, clinical findings suggest that terlipressin can be quite effective in preventing variceal bleeding or in improving renal function, including sodium and water retention in cirrhosis, provided it is used as a single administration or in combination with albumin administration $[34,35]$. In these conditions, terlipressin effects mediated through $\mathrm{V} 1$ receptors seem to overcome the V2 induced deleterious effect in HRS clinical setting. Nevertheless, some caution in its administration mode should be kept in mind, and favouring a continuous intravenous injection instead of bolus injection if repeated boluses are needed [25]. 

(O)AVP
(ם)LVP

$(\Delta)$ Terlipressin
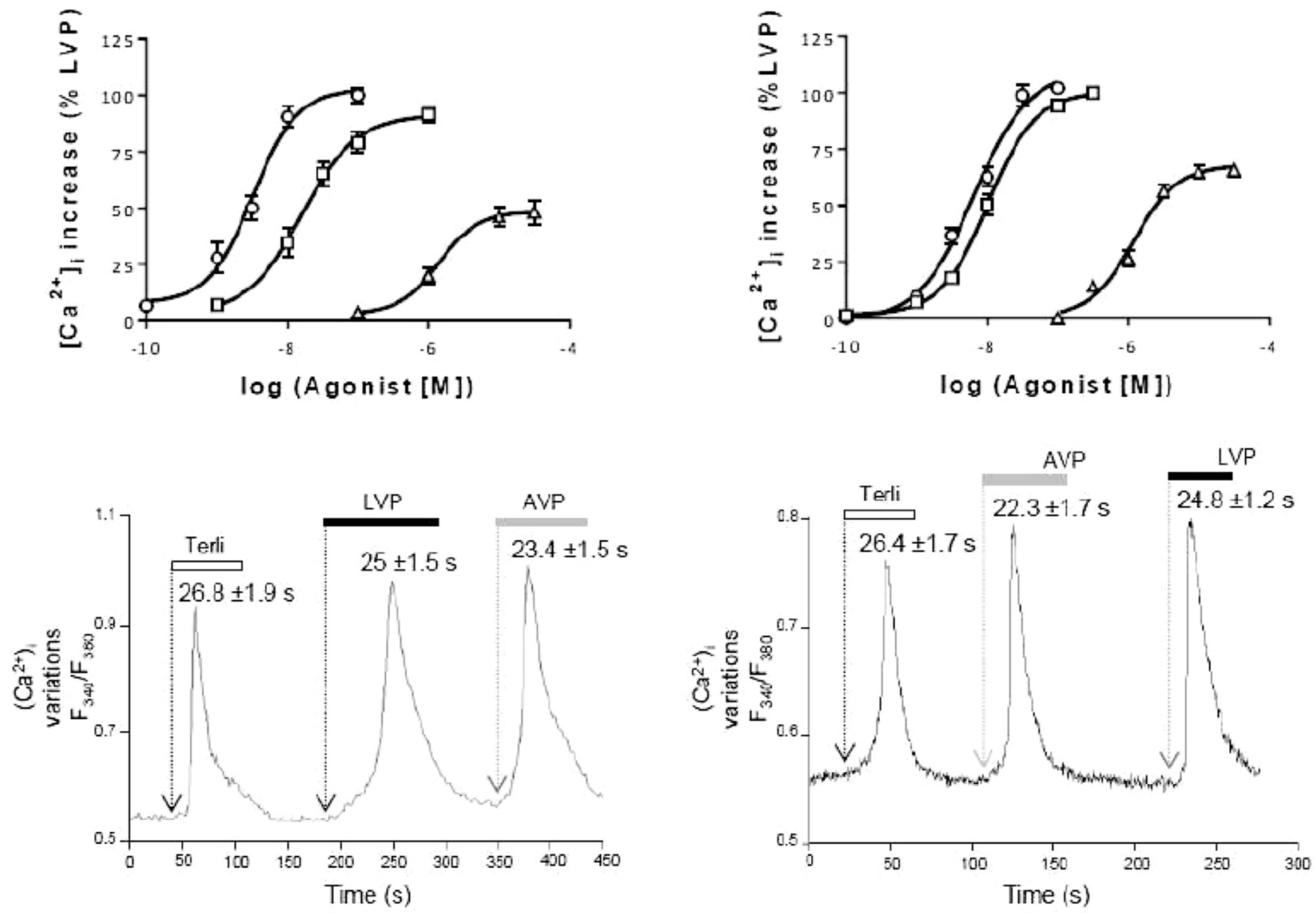

Fig. 4. Effects of AVP, LVP and terlipressin on intracellular calcium variations in rat and human aortic cultured VSMCs.

Intracellular calcium $\left[\mathrm{Ca}^{2+}\right]_{\mathrm{i}}$ increases induced by AVP, LVP or terlipressin were measured in rat aortic and human uterine artery VSMCs as described under methods. Data were averaged (at least 20-25 cells per recorded field/coverglass). One field, chosen randomly, was recorded in each coverglass, $n$ represented the number of cell cultures. Upper panels: Graphs summarize the $\left[\mathrm{Ca}^{2+}\right]_{\mathrm{i}}$ increases in response to increasing concentrations of AVP, LVP and terlipressin. Data, expressed as percentage of the response induced by LVP $\left(10^{-6} \mathrm{M}\right)$ corresponded to the variation of fluorescence ratio for one randomly chosen cellular field containing approximately 20 cells. Results are expressed as\% of maximal LVP stimulation. Values represent the mean \pm SEM of 5 experiments each performed in triplicate. Maximal LVP and terlipressin responses were significantly different between rat and human cells $(\mathrm{P}<0.001)$.

Lower panels: Representative recordings illustrating the time course variations of fluorescence ratio in one individual cell after successive additions of maximally active concentrations of terlipressin $\left(10^{-5} \mathrm{M}\right)$, AVP $\left(10^{-7} \mathrm{M}\right)$ and LVP $\left(10^{-6} \mathrm{M}\right)$. Values represented time to peak for individual responding cells and were averaged on 5 experiments performed in triplicate.

Results obtained shown that, as expected, terlipressin induces $\left[\mathrm{Ca}^{2+}\right]_{\mathrm{i}}$ increase. Moreover its rapid effect, similar to those of LVP, validates a direct action of terlipressin on the $\mathrm{V} 1 \mathrm{~A}$ receptor.

The use of more specific V1A agonists is currently under investigation. Further studies would help to state whether these agonists are preferable to terlipressin in HRS, or to alternative treatments $[36,37]$.

\subsection{Study limitations}

In vitro experimental conditions are quite different from clinical situations in which vasopressin agonists are prescribed. Pharmacological characterization makes experimental models mandatory. Although we can only speculate on the possible consequences of the non-specificity of terlipressin on vasopressin receptors from our in vitro study, these results warrant consideration.

In this study, we have assessed the pharmacological profile of terlipressin, i.e. 3 glycine-vasopressin per se. How the effects of the drug evolve during its slow conversion into LVP in the blood, through the generation of 2 , then 1 glycine -LVP remains to be established. However, our results obtained both on primary cultured cells and on heterologous transfected cells clearly demonstrate that terlipressin is definitively not a V1A selective agonist. Safety reports on terlipressin have mentioned mainly cardiovascular V1-R related effects like peripheral ischaemia, skin necrosis, myocardial infarction and bradycardia, but the second most frequently reported adverse effect is hyponatraemia, a result of V2-R agonism [38]. These adverse effects are now explained by the pharmacological profile reported in the present study.

\section{Conclusions}

Terlipressin is not only a hV1A-R agonist, but also a nearly hV2$\mathrm{R}$ and hV1B-R full agonist. Beyond its vascular effects, terlipressin may have direct renal effects that should be kept in mind during clinical treatment. More specific V1A agonists could be useful to obtain a safer, predictable and specific V1A agonist effect. However, whether the V1A specificity may enhance vasopressin agonist efficiency in HRS deserves further investigation. 


\section{Conflict of interest}

No conflict of interest for any author

\section{Funding}

The study was granted by INSERM, CNRS and Fondation de France.

\section{Acknowledgements}

Special thanks to Nathalie Galeotti for her contribution to the mass spectrometry experiments and Claudine Serradeil LeGal (Sanofi laboratory, Toulouse, France) for her generous gift of SR49059.

\section{References}

[1] J. Uriz, P. Ginès, A. Cárdenas, P. Sort, W. Jiménez, J.M. Salmerón, et al., Terlipressin plus albumin infusion: an effective and safe therapy of hepatorenal syndrome, J. Hepatol. 33 (2000) 43-48.

[2] L.L. Gluud, K. Christensen, E. Christensen, A. Krag, Systematic review of randomized trials on vasoconstrictor drugs for hepatorenal syndrome, Hepatology (Baltimore, Md.) 51 (2010) 576-584, http://dx.doi.org/10.1002/ hep.23286.

[3] M.L. Forsling, L.A. Aziz, M. Miller, R. Davies, B. Donovan, Conversion of triglycylvasopressin to lysine-vasopressin in man, J. Endocrinol. 85 (1980) 237-244.

[4] G. Nilsson, P. Lindblom, M. Ohlin, R. Berling, E. Vernersson, Pharmacokinetics of terlipressin after single i.v. doses to healthy volunteers, Drugs Exp. Clin. Res. 16 (1990) 307-314.

[5] G. Romero, D. Kravetz, J. Argonz, M. Bildozola, A. Suarez, R. Terg, Terlipressin is more effective in decreasing variceal pressure than portal pressure in cirrhotic patients, J. Hepatol. 32 (2000) 419-425.

[6] R. Moreau, F. Durand, T. Poynard, C. Duhamel, J.-P. Cervoni, P. Ichaï, et al., Terlipressin in patients with cirrhosis and type 1 hepatorenal syndrome: a retrospective multicenter study, Gastroenterology 122 (2002) 923-930.

[7] Z. Azam, S. Hamid, W. Jafri, M. Salih, Z. Abbas, S. Abid, et al., Short course adjuvant terlipressin in acute variceal bleeding: a randomized double blind dummy controlled trial, J. Hepatol. 56 (2012) 819-824, http://dx.doi.org/10. 1016/j.jhep.2011.11.019.

[8] V. Singh, S. Ghosh, B. Singh, P. Kumar, N. Sharma, A. Bhalla, et al., Noradrenaline vs terlipressin in the treatment of hepatorenal syndrome: a randomized study, J. Hepatol. 56 (2012) 1293-1298, http://dx.doi.org/10. 1016/j.jhep.2012.01.012.

[9] European Association for the Study of the Liver, EASL clinical practice guidelines on the management of ascites, spontaneous bacterial peritonitis, and hepatorenal syndrome in cirrhosis, J. Hepatol. 53 (2010) 397-417, http:// dx.doi.org/10.1016/j.jhep.2010.05.004.

[10] E. Rodríguez, C. Elia, E. Solà, R. Barreto, I. Graupera, A. Andrealli, et al., Terlipressin and albumin for type-1 hepatorenal syndrome associated with sepsis, J. Hepatol. 60 (2014) 955-961, http://dx.doi.org/10.1016/j.jhep.2013. 12.032.

[11] I. Noguera, P. Medina, G. Segarra, M.C. Martínez, M. Aldasoro, J.M. Vila, et al., Potentiation by vasopressin of adrenergic vasoconstriction in the rat isolated mesenteric artery, Br. J. Pharmacol. 122 (1997) 431-438, http://dx.doi.org/10. 1038/sj.bjp.0701397.

[12] M. Scharte, J. Meyer, H. Van Aken, H.G. Bone, Hemodynamic effects of terlipressin (a synthetic analog of vasopressin) in healthy and endotoxemic sheep, Crit. Care Med. 29 (2001) 1756-1760.

[13] F. Ryckwaert, A. Virsolvy, A. Fort, B. Murat, S. Richard, G. Guillon, et al., Terlipressin, a provasopressin drug exhibits direct vasoconstrictor properties: consequences on heart perfusion and performance, Crit. Care Med. 37 (2009) 876-881, http://dx.doi.org/10.1097/CCM.0b013e31819b8199.

[14] O. Baraldi, C. Valentini, G. Donati, G. Comai, V. Cuna, I. Capelli, et al., Hepatorenal syndrome: update on diagnosis and treatment, World J. Nephrol. 4 (2015) 511-520, http://dx.doi.org/10.5527/wjn.v4.i5.511.

[15] J.F. Quignard, F. Ryckwaert, B. Albat, J. Nargeot, S. Richard, A novel tetrodotoxin-sensitive $\mathrm{Na}+$ current in cultured human coronary myocytes, Circ. Res. 80 (1997) 377-382.

[16] N. Delcourt, P. Jouin, J. Poncet, E. Demey, E. Mauger, J. Bockaert, et al., Difference in mass analysis using labeled lysines (DIMAL-K): a new, efficient proteomic quantification method applied to the analysis of astrocytic secretomes, Mol. Cell Proteomics 4 (2005) 1085-1094, http://dx.doi.org/10. 1074/mcp.M500040-MCP200.

[17] S. Derick, L.L. Cheng, M.J. Voirol, S. Stoev, M. Giacomini, N.C. Wo, et al., [1-deamino-4-cyclohexylalanine] arginine vasopressin: a potent and specific agonist for vasopressin V1b receptors, Endocrinology 143 (2002) 4655-4664, http://dx.doi.org/10.1210/en.2002-220363.
[18] B. Murat, D. Devost, M. Andrés, J. Mion, V. Boulay, M. Corbani, et al., V1b and CRHR1 receptor heterodimerization mediates synergistic biological actions of vasopressin and CRH, Mol. Endocrinol. (Baltimore, Md.) 26 (2012) 502-520, http://dx.doi.org/10.1210/me.2011-1202.

[19] A. Virsolvy, C. Farah, N. Pertuit, L. Kong, A. Lacampagne, C. Reboul, et al., Antagonism of Nav channels and $\alpha 1$-adrenergic receptors contributes to vascular smooth muscle effects of ranolazine, Sci. Rep. 5 (2015) 17969, http:// dx.doi.org/10.1038/srep17969.

[20] C. Barberis, D. Morin, T. Durroux, B. Mouillac, R. Seyer, et al., Molecular pharmacology of AVP and OT receptors and therapeutic potential, Drug News Perspect. 12 (1999) 279-292.

[21] R. Marir, A. Virsolvy, K. Wisniewski, J. Mion, D. Haddou, E. Galibert, et al., Pharmacological characterization of FE 201874, the first selective high affinity rat V1A vasopressin receptor agonist, Br. J. Pharmacol. 170 (2013) 278-292, http://dx.doi.org/10.1111/bph.12249.

[22] G. Guillon, A. Pena, B. Murat, S. Derick, M. Trueba, M.A. Ventura, et al., Position 4 analogues of [deamino-Cys(1)] arginine vasopressin exhibit striking species differences for human and rat V(2)/V(1b) receptor selectivity, J. Pept. Sci. Off. Publ. Eur. Pept. Soc. 12 (2006) 190-198, http://dx.doi.org/10.1002/psc.710.

[23] M.E. Newman, Vasopressin inhibits cyclic AMP accumulation and adenylate cyclase activity in cerebral preparations, FEBS Lett. 181 (1985) 203-206, http://dx.doi.org/10.1016/0014-5793(85)80260-X.

[24] J. Medel, G. Boccara, E. Van de Steen, M. Bertrand, G. Godet, P. Coriat, Terlipressin for treating intraoperative hypotension: can it unmask myocardial ischemia? Anesth. Analg. 93 (2001) 53-55, TOC.

[25] M. Cavallin, S. Piano, A. Romano, S. Fasolato, A.C. Frigo, G. Benetti, et al., Terlipressin given by continuous intravenous infusion versus intravenous boluses in the treatment of hepatorenal syndrome: a randomized controlled study, Hepatology (Baltimore, Md.) 63 (2016) 983-992, http://dx.doi.org/10. 1002/hep.28396.

[26] A. Morelli, C. Ertmer, S. Rehberg, M. Lange, A. Orecchioni, V. Cecchini, et al., Continuous terlipressin versus vasopressin infusion in septic shock (TERLIVAP): a randomized, controlled pilot study, Crit. Care Lond. Engl. 13 (2009) R130, http://dx.doi.org/10.1186/cc7990.

[27] X. Xiao, Y. Zhu, D. Zhen, X.M. Chen, W. Yue, L. Liu, et al., Beneficial and side effects of arginine vasopressin and terlipressin for septic shock, J. Surg. Res. 195 (2015) 568-579, http://dx.doi.org/10.1016/j.jss.2015.02.022.

[28] E. Solà, S. Lens, M. Guevara, M. Martín-Llahí, C. Fagundes, G. Pereira, et al., Hyponatremia in patients treated with terlipressin for severe gastrointestinal bleeding due to portal hypertension, Hepatology (Baltimore, Md.) 52 (2010) 1783-1790, http://dx.doi.org/10.1002/hep.23893.

[29] A. Krag, F. Bendtsen, E.B. Pedersen, N.-H. Holstein-Rathlou, S. Møller, Effects of terlipressin on the aquaretic system: evidence of antidiuretic effects, Am. J. Physiol. Renal Physiol. 295 (2008) F1295-1300, http://dx.doi.org/10.1152/ ajprenal.90407.2008.

[30] S. Jard, R.C. Gaillard, G. Guillon, J. Marie, P. Schoenenberg, A.F. Muller, et al., Vasopressin antagonists allow demonstration of a novel type of vasopressin receptor in the rat adenohypophysis, Mol. Pharmacol. 30 (1986) 171-177.

[31] R.W. Hunter, J.R. Ivy, M.A. Bailey, Glucocorticoids and renal Na+transport: implications for hypertension and salt sensitivity, J. Physiol. 592 (2014) 1731-1744, http://dx.doi.org/10.1113/jphysiol.2013.267609.

[32] P. Ginès, F. Wong, H. Watson, S. Milutinovic, L.R. del Arbol, D. Olteanu, et al., Effects of satavaptan, a selective vasopressin $\mathrm{V}(2)$ receptor antagonist, on ascites and serum sodium in cirrhosis with hyponatremia: a randomized trial, Hepatology (Baltimore, Md.) 48 (2008) 204-213, http://dx.doi.org/10.1002/ hep.22293.

[33] M.-H. Tsai, Y.-S. Peng, Y.-C. Chen, N.-J. Liu, Y.-P. Ho, J.-T. Fang, et al., Adrenal insufficiency in patients with cirrhosis, severe sepsis and septic shock, Hepatology (Baltimore, Md.) 43 (2006) 673-681, http://dx.doi.org/10.1002/ hep.21101.

[34] A. Krag, S. Møller, J.H. Henriksen, N.-H. Holstein-Rathlou, F.S. Larsen, F. Bendtsen, Terlipressin improves renal function in patients with cirrhosis and ascites without hepatorenal syndrome, Hepatology (Baltimore, Md.) 46 (2007) 1863-1871, http://dx.doi.org/10.1002/hep.21901.

[35] T.D. Boyer, A.J. Sanyal, F. Wong, R.T. Frederick, J.R. Lake, J.G. O'Leary, et al., Terlipressin plus albumin is more effective than albumin alone in improving renal function in patients with cirrhosis and hepatorenal syndrome type 1 , Gastroenterology 150 (2016) 1579-1589, http://dx.doi.org/10.1053/j.gastro. 2016.02.026, e2.

[36] X. He, F. Su, F.S. Taccone, R. Laporte, A.L. Kjølbye, J. Zhang, et al., A selective $\mathrm{V} 1 \mathrm{~A}$ receptor agonist, selepressin, is superior to arginine vasopressin and to norepinephrine in ovine septic shock, Crit. Care Med. 44 (2016) 23-31, http:// dx.doi.org/10.1097/CCM.0000000000001380.

[37] M. Cavallin, P.S. Kamath, M. Merli, S. Fasolato, P. Toniutto, F. Salerno, et al., Terlipressin plus albumin versus midodrine and octreotide plus albumin in the treatment of hepatorenal syndrome: a randomized trial, Hepatol Baltim Md 62 (2015) 567-574, http://dx.doi.org/10.1002/hep.27709.

[38] Australian government. Department of health and Ageing. Therapeutic Goods Administration. Australian Public Assessment Report for terlipressin acetate, November 2012. 